PREPARED FOR THE U.S. DEPARTMENT OF ENERGY, UNDER CONTRACT DE-AC02-76CH03073

PPPL-3784

PPPL-3784

UC-70

Electromagnetic Fluctuations during Fast Reconnection in a Laboratory Plasma

by

Hantao Ji, Stephen Terry, Masaaki Yamada, Russell Kulsrud, Aleksey Kuritsyn, and Yang Ren

February 2003

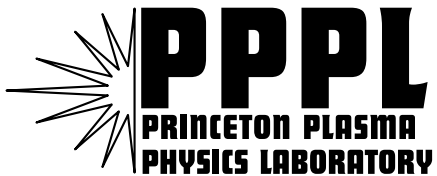

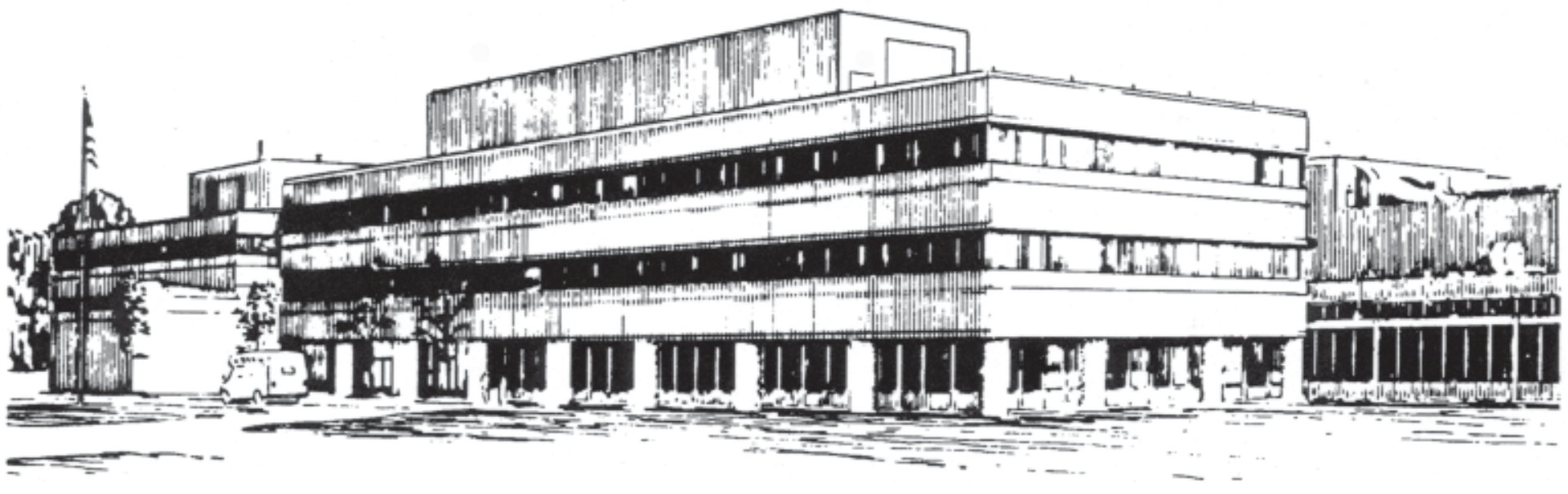

PRINCETON PLASMA PHYSICS LABORATORY PRINCETON UNIVERSITY, PRINCETON, NEW JERSEY 


\section{PPPL Reports Disclaimer}

This report was prepared as an account of work sponsored by an agency of the United States Government. Neither the United States Government nor any agency thereof, nor any of their employees, makes any warranty, express or implied, or assumes any legal liability or responsibility for the accuracy, completeness, or usefulness of any information, apparatus, product, or process disclosed, or represents that its use would not infringe privately owned rights. Reference herein to any specific commercial product, process, or service by trade name, trademark, manufacturer, or otherwise, does not necessarily constitute or imply its endorsement, recommendation, or favoring by the United States Government or any agency thereof. The views and opinions of authors expressed herein do not necessarily state or reflect those of the United States Government or any agency thereof.

\section{Availability}

This report is posted on the U.S. Department of Energy's Princeton Plasma Physics Laboratory Publications and Reports web site in Fiscal Year 2003. The home page for PPPL Reports and Publications is: http://www.pppl.gov/pub_report/

DOE and DOE Contractors can obtain copies of this report from:

U.S. Department of Energy

Office of Scientific and Technical Information

DOE Technical Information Services (DTIS)

P.O. Box 62

Oak Ridge, TN 37831

Telephone: (865) 576-8401

Fax: (865) 576-5728

Email: reports@adonis.osti.gov

This report is available to the general public from:

National Technical Information Service

U.S. Department of Commerce

5285 Port Royal Road

Springfield, VA 22161

Telephone: $1-800-553-6847$ or

(703) $605-6000$

Fax: (703) 321-8547

Internet: http://www.ntis.gov/ordering.htm 


\title{
Electromagnetic Fluctuations during Fast Reconnection in a Laboratory Plasma
}

\author{
Hantao Ji, Stephen Terry, Masaaki Yamada, \\ Russell Kulsrud, Aleksey Kuritsyn, and Yang Ren \\ Princeton Plasma Physics Laboratory, \\ Princeton University, P.O. Box 451, Princeton, NJ 08543
}

(Dated: January 29, 2003)

\begin{abstract}
Clear evidence for a positive correlation is established between the magnitude of magnetic fluctuations in the lower-hybrid frequency range and enhancement of reconnection rates in a wellcontrolled laboratory plasma. The fluctuations belong to the right-hand polarized whistler wave branch, propagating obliquely to the reconnecting magnetic field, with a phase velocity comparable to the relative drift velocity between electrons and ions. The short coherence length and large variation along the propagation direction indicate their strongly nonlinear nature in three dimensions.
\end{abstract}

PACS numbers: 52.35.Vd, 52.35.Qz, 52.35.Ra, 52.72.+v 
Magnetic reconnection [e.g. 1] plays an important role in determining the evolution of magnetic topology in relaxation processes in high-temperature laboratory plasmas, magnetospheric substorms, solar flares, and more distant astrophysical plasmas. Often, magnetic reconnection is invoked to explain the observed rapid release of magnetic energy in these highly conducting plasmas. A central question of magnetic reconnection concerns why the observed reconnection rates are much faster than predictions by the Sweet-Parker model $[2,3]$ based on magnetohydrodynamics (MHD) with the classical Spitzer resistivity. In this two-dimensional (2D) model, the infinitely small resistivity causes magnetic field to dissipate only in very thin current sheets, which impede the outflow of mass leading to significantly slow reconnection rates. The subsequently proposed Petschek model [4] is based on standing slow shocks to open up the outflow channel allowing larger mass flows thus the faster reconnection rates. However, it has been shown later $[5,6]$ that the Petschek solution is not compatible with uniform or smooth resistivity profiles. On the other hand, perhaps not surprisingly, the plasma resistivity can be enhanced due to microinstabilities which are often active only in the reconnection region where plenty of free energy exists in the form of a large relative drift between ions and electrons and large inhomogeneities in pressure and magnetic field. This anomalous resistivity not only can broaden the current sheet to increase the mass flow and the reconnection rate in the context of the Sweet-Parker model [7] but also its localization is able to open up the outflow channel for the fast reconnection [7-9]. Alternatively, a recent theory [10] attempts to explain fast reconnection rates based on nondissipative terms, notably the Hall term, in the generalized Ohm's law in a 2D and laminar fashion.

The underlying microinstabilities [1] for the resistivity enhancement have been considered to be predominantly electrostatic in nature due to their effectiveness in wave-particle interactions. The primary candidate is the Lower-Hybrid Drift Instability (LHDI) [11], which has been frequently observed in space plasmas $[12,13]$. Recently this instability has been studied in direct relation with reconnection in laboratory [14] and in space $[15,16]$. The main conclusion of these studies is that the LHDI is active only at the low- $\beta$ edge region of current sheet, but not at the high- $\beta$ central region, where the resistivity needs to be enhanced for fast reconnection. This conclusion is consistent with an earlier theory [17] which showed stabilization of LHDI by finite plasma $\beta$, and also with more recent numerical simulations $[18,19]$. 
By contrast, much less attention has been paid to electromagnetic fluctuations and their relation with reconnection, although they have been also regularly observed in space [12]. In this Letter, we report the first clear experimental evidence for electromagnetic fluctuations in the lower-hybrid frequency range during fast reconnection in a well-controlled laboratory plasma, Magnetic Reconnection Experiment (MRX) [20]. The observed waves are identified as right-hand polarized whistler waves propagating obliquely to the magnetic field. Earlier laboratory experiments [21] on reconnection have indicated evidence for high-frequency electromagnetic fluctuations in the electron MHD regime where only electrons are magnetized, but their roles in the reconnection process were unclear. On the other hand, most plasmas of interest for the reconnection problem are well into the MHD regime where ions are also magnetized at least outside of the reconnection region, as in MRX. In one case, magnetic fluctuations have been interpreted as byproducts of LHDI in space plasmas [15].

In the MRX, magnetic reconnection is driven by reducing currents in two flux cores whose toroidally symmetric shape ensures the global 2D geometry, as illustrated in Fig.1. Oppositely directed field lines in the $Z$ direction are pulled together radially, forming a current sheet flowing along the $\theta$ direction. All essential parameters needed for characterizing reconnection are measured by an extensive set of diagnostics [20]. In a previous quantitative study [22], it was shown that the observed reconnection rates can be explained by a modified Sweet-Parker model including an effective resistivity, determined experimentally by $\eta^{*} \equiv E_{\theta} / j_{\theta}$ where $E_{\theta}$ and $j_{\theta}$ are toroidal reconnecting electric field and current density, respectively. In highly collisional plasmas, $\eta^{*}$ is very close to the classical Spitzer perpendicular resistivity $\eta_{\text {Spitzer }}[23]$ while $\eta^{*} \gg \eta_{\text {Sptizer }}$ when the collisionality is reduced [22], in correlation with strong nonclassical ion heating [24]. High-frequency fluctuations have been measured in MRX in order to explore the possibility of the resistivity enhancement due to microinstabilities in low collisionality regimes. Although the LHDI was identified at the low- $\beta$ edge region of the current sheet, electrostatic fluctuations did not correlate well with the reconnection process in their temporal and spatial behavior as well as their collisionality dependence, leading to a conclusion that the electrostatic fluctuations do not play an essential role in the fast reconnection in $\operatorname{MRX}[14,25]$.

The experimental results reported here focus on electromagnetic fluctuations, which do correlate well with fast reconnection. The main diagnostics used are based on four small magnetic pickup coils mounted inside an electrostatically shielded glass tube. All three com- 


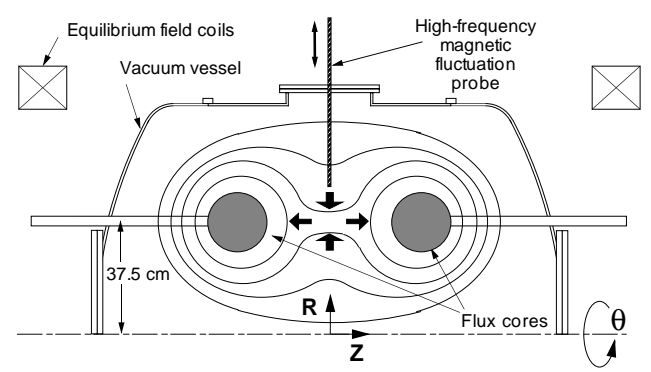

FIG. 1: Experimental setup in MRX for magnetic reconnection which is induced by reducing coil currents in the "flux cores". Oppositely directed magnetic field lines is driven towards each other to form a current sheet flowing in the $\theta$ direction. Inflow and outflow are in the radial (R) and axial (Z) directions, respectively.

ponents of magnetic field are measured at almost the same location. The probe outputs are fed into a miniature circuit board, which houses four amplifiers embedded in the probe shaft near the tip in order to provide noise immunity and impedance matching. The integrated bandwidth is up to $30 \mathrm{MHz}$.

Typical raw signals are shown in Fig.2 during a single discharge when reconnection is induced. High-frequency fluctuations appear in all three components of magnetic field right after $t=260 \mu$ s when the current sheet is formed, and persist as long as the reconnection goes on. Spectrograms, which display the fluctuation power in the time-frequency domain, are shown in the right panels of Fig.2. It is seen that discrete peaks exist in the lower-
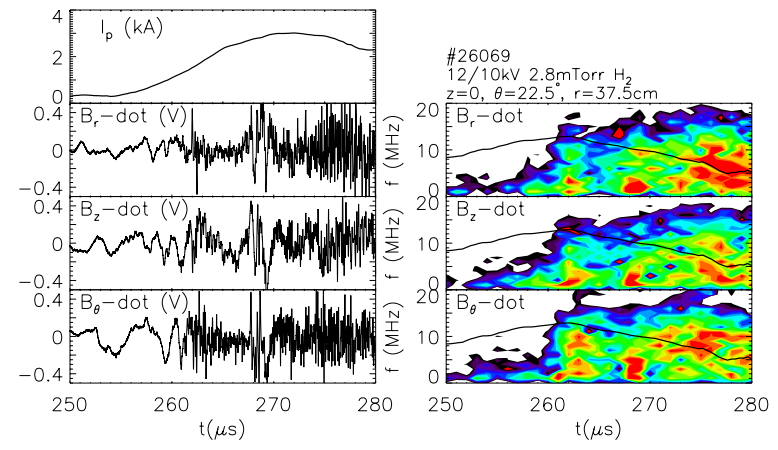

FIG. 2: Traces of typical raw signals during reconnection represented by plasma current (top left panel). Spectrograms of each signal are also shown on the right panels where fluctuation powers are color-coded (decreasing power by order of red, yellow, green, blue and white) in the time-frequency domain. The black lines indicate local $f_{\mathrm{LH}}$. 
hybrid frequency range and they tend to also vary accordingly with the changes in the local

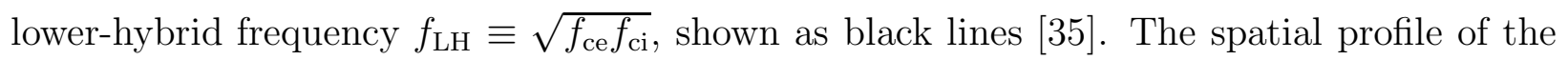
total fluctuation amplitude $|\widetilde{B}|$ is plotted in Fig.3. The fluctuations have large amplitudes consistently near the current sheet center with peak $|\widetilde{B}| / B_{\text {up }}$ up to $5 \%$, where $B_{\text {up }}$ is the upstream reconnecting magnetic field. It should be noted that both temporal behavior and spatial behavior of the magnetic fluctuations are in sharp contrast with the electrostatic fluctuations $[14,25]$.

In order to identify the observed electromagnetic waves, it is crucial to measure their propagation characteristics. Two techniques have been employed for this purpose. The first one is called the hodogram technique [26], which is based on the tip trajectories of a fluctuating magnetic field vector measured at a single point in space. For a given frequency, the condition $\nabla \cdot \widetilde{\boldsymbol{B}}=0$ can be translated to $\boldsymbol{k} \cdot \widetilde{\boldsymbol{B}}=0$ where $\boldsymbol{k}$ is the dominant wavenumber vector, the direction of $\boldsymbol{k}$ can be determined by the right-hand rule $\widetilde{\boldsymbol{B}}\left(t_{0}\right) \times \widetilde{\boldsymbol{B}}\left(t_{0}+\delta t\right)$ when $f \gg f_{\text {ci }}$. Power spectra of the fluctuating magnetic field can be constructed in the domain of frequency and the angle between $\boldsymbol{k}$ and the background $\boldsymbol{B}_{0}$, as shown in Fig.4(left). It is seen that in the low frequencies, $\boldsymbol{k}$ has a rather large angle $\left(\sim 60^{\circ}\right)$ to $\boldsymbol{B}_{0}$ with a broad spread while in higher frequencies, $\boldsymbol{k}$ has a rather small angle $\left(\sim 30^{\circ}\right)$ to $\boldsymbol{B}_{0}$ with a narrow spread. In addition, since the $\boldsymbol{k}$ vector has only a small radial component (not shown), $\boldsymbol{k}$ remains in the $Z-\theta$ plane. Therefore, it is concluded that the observed magnetic fluctuations are right-hand polarized whistler-like waves propagating obliquely to the magnetic field while staying within the current sheet.

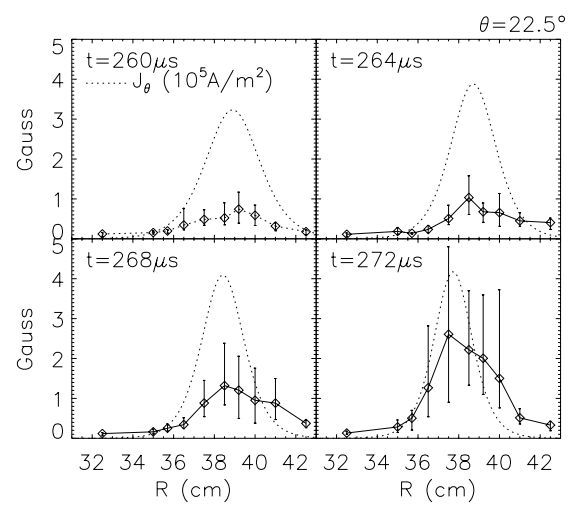

FIG. 3: Radial profile of total amplitude of magnetic fluctuations with $f \geq 1 \mathrm{MHz}$ at four time points. The current density profiles (dotted lines) are also shown for reference. 

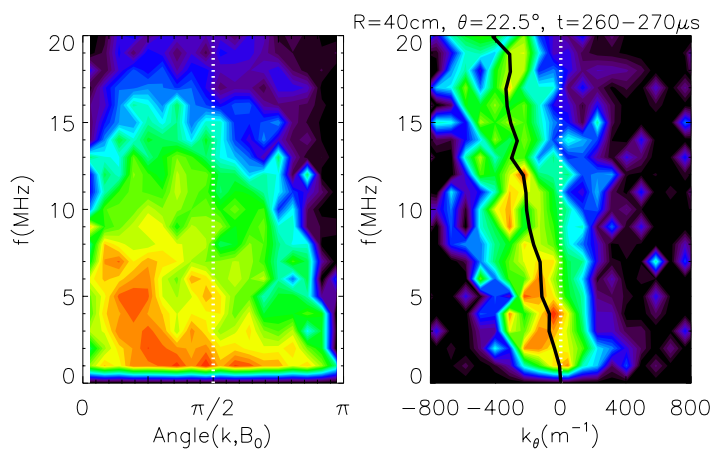

FIG. 4: Power spectra (color-coded as in Fig.2) for $\widetilde{B}$ in the domain of frequency and the angle between dominant $\boldsymbol{k}$ and $\boldsymbol{B}_{0}$ (left) and for $\widetilde{B}_{Z}$ in the domain of frequency and $k_{\theta}$ (right) at $R=40 \mathrm{~cm}$.

While the direction of $\boldsymbol{k}$ is determined by the hodogram technique, its magnitude or the wavelength is determined by a second technique using the phase shift measured between two spatial points. Two glass tubes, each containing a pickup coil, are mounted to a probe shaft with a distance of $7 \mathrm{~mm}$ between their axes. Each signal is fed to a single-channel version of the miniature in-shaft amplifier [14]. The phase shift along the $\theta$ or $Z$ direction is measured to construct power spectra in the domain of frequency and $k_{\theta}$ [Fig.4(right)] or $k_{Z}$ (not shown). It is found that the magnetic fluctuations propagate mainly in the $-\theta$ direction, along which electrons drift, but not in the $Z$ direction. The phase velocity, $V_{\mathrm{ph}}=(3.4 \pm 0.8) \times 10^{5} \mathrm{~m} / \mathrm{s}$ calculated from the slope of the black line in Fig.4(right), is reasonably consistent with the relative drift velocity $V_{\mathrm{d}} \equiv j_{\theta} /$ en $=(2.5 \pm 0.9) \times 10^{5} \mathrm{~m} / \mathrm{s}$, where $n$ is the plasma density.

The measurements of the propagation characteristics described above are made at the outer edge of the current sheet $(R=40 \mathrm{~cm})$ where the fluctuations have only moderate amplitudes (see Fig.3) and stay relatively coherent within the probe separation. At the current sheet center where the fluctuation amplitude peaks, the measurements of $\boldsymbol{k}$ have not been so successful due to extremely short coherence lengths. The coherence $\gamma \equiv\left|\widetilde{B}_{1} \widetilde{B}_{2}^{*}\right| /\left(\left|\widetilde{B}_{1}\right|\left|\widetilde{B}_{2}\right|\right)$ between signals $\widetilde{B}_{1}$ and $\widetilde{B}_{2}$ rapidly decreases to the noise level when the separation is larger than $0.5-1.5 \mathrm{~cm}$, suggesting their strongly nonlinear nature. (For comparison, the wavelength measured at $R=40 \mathrm{~cm}$ in the same $\theta$ direction is $\sim 5 \mathrm{~cm}$ for $f=10 \mathrm{MHz}$.) Furthermore, it is found that the fluctuation amplitude varies substantially along the $\theta$ (current) direction, breaking the 2D axisymmetry of the current sheet and consequently the reconnection 
process. Further detailed measurements indicate that the fluctuation amplitude tends to correlate with the local $V_{\mathrm{d}}$.

The next important question concerns how the observed magnetic fluctuations are related to the resistivity enhancement, and thus the fast reconnection process in MRX. It is consistently found that the fluctuation amplitudes are sensitive to plasma density or equivalently the collisionality. For example, when the density is reduced from $\sim 5 \times 10^{19} \mathrm{~m}^{-3}$ to $\sim 2 \times 10^{18} \mathrm{~m}^{-3},\left|\widetilde{B}_{Z}\right|$ increases from $0.1 \mathrm{G}$ (close to the noise level for the measurements) to $\sim 1 \mathrm{G}$, as shown in Fig.5. Since the resistivity enhancement also strongly depends on the plasma collisionality [22] (Fig.5), a clear positive correlation between magnetic fluctuations and resistivity enhancement is established, as shown in Fig.6 [36].

Given the experimental data described above, it is clear that the observed electromagnetic waves are caused by microinstabilities driven by free energy stored in the reconnecting current sheet, in the form of large $V_{\mathrm{d}}$ and/or large inhomogeneities. Since essentially no electrostatic fluctuations are detected concurrently, the observed magnetic fluctuations cannot be simply byproducts of LHDI, which is driven by inhomogeneities, propagating primarily perpendicular to the field. In addition, magnetic fluctuations due to LHDI are dominated by their component along $\boldsymbol{B}_{0}$ while experimentally all three components have roughly the same amplitude (see Fig.2). The waves also cannot be described as whistler waves driven by electron temperature anisotropy [27], which propagate mainly along $\boldsymbol{B}_{0}$.

Many observed key features of the magnetic fluctuations, however, are consistent with the so-called Modified Two-Stream Instability (MTSI) in the high- $\beta$ limit $[11,28-31]$. In contrast to LHDI, MTSI is driven only by a large relative drift across the magnetic field in homogeneous plasmas. In the low- $\beta$ limit, both LHDI and MTSI behave similarly [32].

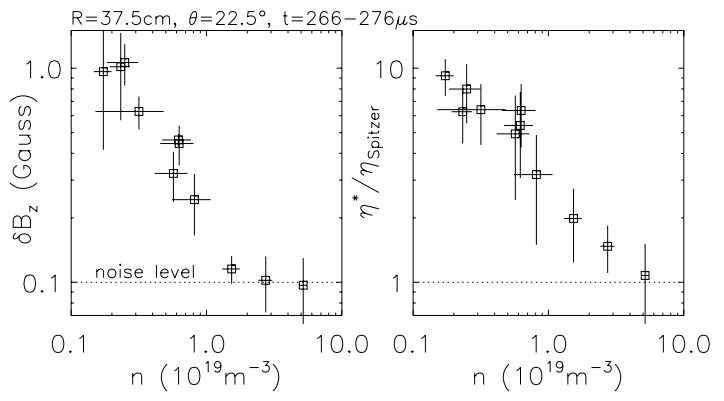

FIG. 5: Density dependence of $\widetilde{B}_{Z}$ amplitude at the current sheet center and resistivity enhancement. 


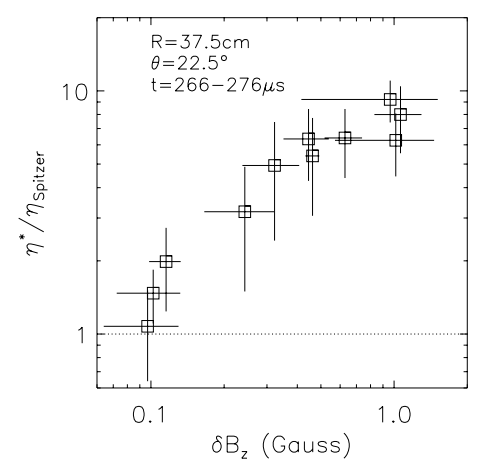

FIG. 6: Resistivity enhancement versus fluctuation amplitude of $\widetilde{B}_{Z}$ at the current sheet center.

When $\beta$ is large ( $\gtrsim 1)$, LHDI is stabilized [17] while MTSI remains unstable but the Alfvén speed $V_{\mathrm{A}}$ replaces the ion sound (or thermal) speed as the critical speed for $V_{\mathrm{d}}$. The resultant waves are largely electromagnetic and right-hand polarized whistler-like waves propagating obliquely to the field with $V_{\mathrm{ph}} \sim V_{\mathrm{d}}$ [28]. Under certain conditions similar to those at the current sheet in MRX, such as $\beta \gtrsim 1$ and $V_{\mathrm{d}} / V_{\mathrm{A}} \gtrsim 5$, the waves are unstable only at certain propagation angles to the field [30,31]. However, the discrete peaks in the frequency spectra (Fig.2) cannot be explained by these theories, which were based on the slab geometry. Global eigenmode calculations taking into account the boundary conditions, as have been done for LHDI [33, 34], may explain the discrete frequency peaks.

Quantitative estimates of resistivity enhancement due to these fully nonlinear waves as described above are not straightforward. Nonetheless, examinations of each term in the generalized Ohm's law reveal that the turbulent Hall term due to the magnetic fluctuations could be sufficiently large to balance the reconnecting electric field if the measured coherence lengths are used as the perpendicular scale length. Other candidates, such as off-diagonal terms in the pressure tensor, can also possibly provide required effective friction between electrons and ions as a result of wave-particle interactions.

In summary, a detailed experimental study in MRX has established, for the first time, a clear and positive correlation between magnetic fluctuations in the lower-hybrid frequency range and fast reconnection in the low-collisionality regimes. The waves have been identified as right-hand polarized whistler waves, propagating obliquely to the reconnecting field, with a phase velocity comparable to the relative drift velocity. These waves are consistent with the modified two-stream instability driven by large drift speeds compared to the Alfvén speed in high- $\beta$ plasmas. The short coherence length and large variation along the 
propagation direction indicate their strongly nonlinear nature. Quantitative understanding of the effects of these waves on the resistivity enhancement, and thus reconnection rates, requires further experimental efforts to directly measure relevant terms in the generalized Ohm's law. Theories and numerical studies of linear and nonlinear characteristics of currentdriven microinstabilities using proper models and boundary conditions in 3D should provide much-needed physical insight.

The authors are grateful to D. Cylinder and R. Cutler for their excellent technical support, J. Whitney, K. Shen, and J. Carter for their contributions through a summer program, and T. Carter for useful discussions. This work was jointly supported by DOE, NASA, and NSF.

[1] D. Biskamp, Magnetic reconnection in plasmas (Cambridge University Press, Cambridge, 2000).

[2] P. Sweet, in Electromagnetic Phenomena in Cosmical Physics, edited by B. Lehnert (Cambridge Univ. Press, New York, 1958), p. 123.

[3] E. Parker, J. Geophys. Res. 62, 509 (1957).

[4] H. Petschek, NASA Spec. Pub. 50, 425 (1964).

[5] D. Biskamp, Phys. Fluids 29, 1520 (1986).

[6] D. Uzdensky and R. Kulsrud, Phys. Plasmas 7, 4018 (2000).

[7] R. Kulsrud, Earth Planets Space 53, 417 (2001).

[8] M. Ugai and T. Tsuda, J. Plasma Phys. 17, 337 (1977).

[9] D. Biskamp and E. Schwarz, Phys. Plasmas 8, 4729 (2001).

[10] J. Birn et al., J. Geophys. Res. 106, 3715 (2001).

[11] N. Krall and P. Liewer, Phys. Rev. A 4, 2094 (1971).

[12] D. Gurnett et al., J. Geophys. Res. 81, 6059 (1976).

[13] C. Cattell and F. Mozer, Geophys. Res. Lett. 13, 221 (1986).

[14] T. Carter et al., Phys. Rev. Lett. 88, 015001 (2002).

[15] I. Shinohara et al., J. Geophys. Res. 103, 20365 (1998).

[16] S. Bale et al., Geophys. Res. Lett. 29, to appear (2003).

[17] R. Davidson et al., Phys. Fluids 20, 301 (1977).

[18] R. Horiuchi and T. Sato, Phys. Plasmas 6, 4565 (1999). 
[19] G. Lapenta and J. Brackbill, Phys. Plasmas 9, 1544 (2002).

[20] M. Yamada et al., Phys. Plasmas 4, 1936 (1997).

[21] W. Gekelman and R. Stenzel, J. Geophys. Res. 89, 2715 (1984).

[22] H. Ji et al., Phys. Rev. Lett. 80, 3256 (1998).

[23] F. Trintchouk et al., Phys. Plasmas 10, 319 (2003).

[24] S. Hsu et al., Phys. Rev. Lett. 84, 3859 (2000).

[25] T. Carter et al., Phys. Plasmas 9, 3727 (2002).

[26] J. Urrutia and R. Stenzel, Phys. Plasmas 7, 4457 (2000).

[27] C. Kennel and H. Petschek, J. Geophys. Res. 71, 1 (1966).

[28] D. W. Ross, Phys. Fluids 13, 746 (1970).

[29] J. McBride et al., Phys. Fluids 15, 2367 (1972).

[30] C. Wu et al., Phys. Fluids 26, 1259 (1983).

[31] B. Basu and B. Coppi, J. Geophys. Res. 97, 17033 (1992).

[32] O. Silveira et al., Phys. Rev. E 65, 036407 (2002).

[33] P. Yoon et al., Phys. Plasmas 9, 1526 (2002).

[34] W. Daughton, 2002, private communication.

[35] The local magnetic field (and $f_{\mathrm{LH}}$ ), remains nonzero at the current sheet center due to a residual toroidal field generated during the plasma formation process [20].

[36] The resistivity is calculated by assuming toroidal symmetry of the current sheet [22], which does not necessarily hold true in the discharges used for this study. However, the averaged resistivity obtained by a scan over 20 toroidal angles is reasonably consistent with values based on the axisymmetry assumption provided enough numbers of discharges are used for averaging. 


\section{External Distribution}

Plasma Research Laboratory, Australian National University, Australia

Professor I.R. Jones, Flinders University, Australia

Professor João Canalle, Instituto de Fisica DEQ/IF - UERJ, Brazil

Mr. Gerson O. Ludwig, Instituto Nacional de Pesquisas, Brazil

Dr. P.H. Sakanaka, Instituto Fisica, Brazil

The Librarian, Culham Laboratory, England

Mrs. S.A. Hutchinson, JET Library, England

Professor M.N. Bussac, Ecole Polytechnique, France

Librarian, Max-Planck-Institut für Plasmaphysik, Germany

Jolan Moldvai, Reports Library, MTA KFKI-ATKI, Hungary

Dr. P. Kaw, Institute for Plasma Research, India

Ms. P.J. Pathak, Librarian, Insitute for Plasma Research, India

Ms. Clelia De Palo, Associazione EURATOM-ENEA, Italy

Dr. G. Grosso, Instituto di Fisica del Plasma, Italy

Librarian, Naka Fusion Research Establishment, JAERI, Japan

Library, Plasma Physics Laboratory, Kyoto University, Japan

Research Information Center, National Institute for Fusion Science, Japan

Dr. O. Mitarai, Kyushu Tokai University, Japan

Library, Academia Sinica, Institute of Plasma Physics, People's Republic of China

Shih-Tung Tsai, Institute of Physics, Chinese Academy of Sciences, People's Republic of China

Dr. S. Mirnov, TRINITI, Troitsk, Russian Federation, Russia

Dr. V.S. Strelkov, Kurchatov Institute, Russian Federation, Russia

Professor Peter Lukac, Katedra Fyziky Plazmy MFF UK, Mlynska dolina F-2, Komenskeho Univerzita, SK-842 15 Bratislava, Slovakia

Dr. G.S. Lee, Korea Basic Science Institute, South Korea

Institute for Plasma Research, University of Maryland, USA

Librarian, Fusion Energy Division, Oak Ridge National Laboratory, USA

Librarian, Institute of Fusion Studies, University of Texas, USA

Librarian, Magnetic Fusion Program, Lawrence Livermore National Laboratory, USA

Library, General Atomics, USA

Plasma Physics Group, Fusion Energy Research Program, University of California at San Diego, USA

Plasma Physics Library, Columbia University, USA

Alkesh Punjabi, Center for Fusion Research and Training, Hampton University, USA

Dr. W.M. Stacey, Fusion Research Center, Georgia Institute of Technology, USA

Dr. John Willis, U.S. Department of Energy, Office of Fusion Energy Sciences, USA

Mr. Paul H. Wright, Indianapolis, Indiana, USA 
The Princeton Plasma Physics Laboratory is operated by Princeton University under contract with the U.S. Department of Energy.

\author{
Information Services \\ Princeton Plasma Physics Laboratory \\ P.O. Box 451 \\ Princeton, NJ 08543
}

Phone: 609-243-2750

Fax: 609-243-2751

e-mail: pppl_info@pppl.gov

Internet Address: http://www.pppl.gov 